\title{
Association of age with response to preoperative chemotherapy in patients with muscle-invasive bladder cancer
}

\author{
David D'Andrea ${ }^{1} \cdot$ Peter C. Black ${ }^{2} \cdot$ Homayoun Zargar ${ }^{2,3} \cdot$ Kamran Zargar-Shoshtari $^{4,5} \cdot$ Francesco Soria $^{6}$. \\ Adrian S. Fairey ${ }^{7} \cdot$ Laura S. Mertens $^{8} \cdot$ Colin P. Dinney $^{9} \cdot$ Maria C. Mir $^{10,11} \cdot$ Laura-Maria Krabbe $^{12,13}$. \\ Michael S. Cookson ${ }^{14} \cdot$ Niels-Erik Jacobsen ${ }^{7}$. Jeffrey S. Montgomery ${ }^{15} \cdot$ Nikhil Vasdev $^{16,17} \cdot$ Evan Y. Yu $^{18}$. \\ Evanguelos Xylinas ${ }^{19}$. Nicholas J. Campain ${ }^{20}$. Wassim Kassouf ${ }^{21}$ - Marc A. Dall'Era ${ }^{22}$. Jo-An Seah ${ }^{23}$. \\ Cesar E. Ercole $^{10}$. Simon Horenblas ${ }^{8}$. Srikala S. Sridhar ${ }^{23}$. John S. McGrath ${ }^{20}$. Jonathan Aning ${ }^{20,24}$. \\ Jonathan L. Wright ${ }^{25}$. Andrew C. Thorpe ${ }^{17}$. Todd M. Morgan ${ }^{15}$. Jeff M. Holzbeierlein ${ }^{26}$. Trinity J. Bivalacqua ${ }^{27}$. \\ Scott North ${ }^{28,29}$. Daniel A. Barocas ${ }^{30}$. Yair Lotan ${ }^{12}$. Petros Grivas ${ }^{18,31}$. Andrew J. Stephenson ${ }^{10}$. Jay B. Shah ${ }^{9,32}$. \\ Bas W. van Rhijn ${ }^{8} \cdot$ Siamak Daneshmand $^{33}$. Philippe E. Spiess ${ }^{4} \cdot$ Shahrokh F. Shariat ${ }^{1,12,34,35,36}$
}

Received: 29 April 2021 / Accepted: 19 July 2021 / Published online: 9 August 2021

(c) The Author(s) 2021

\begin{abstract}
Purpose To assess the association of patient age with response to preoperative chemotherapy in patients with muscle-invasive bladder cancer (MIBC).

Materials and methods We analyzed data from 1105 patients with MIBC. Patients age was evaluated as continuous variable and stratified in quartiles. Pathologic objective response (pOR; ypT0-Ta-Tis-T1N0) and pathologic complete response (pCR; ypT0N0), as well survival outcomes were assessed. We used data of 395 patients from The Cancer Genome Atlas (TCGA) to investigate the prevalence of TCGA molecular subtypes and DNA damage repair (DDR) gene alterations according to patient age.

Results pOR was achieved in $40 \%$ of patients. There was no difference in distribution of pOR or pCR between age quartiles. On univariable logistic regression analysis, patient age was not associated with pOR or pCR when evaluated as continuous variables or stratified in quartiles (all $p>0.3$ ). Median follow-up was 18 months (IQR 6-37). On Cox regression and competing risk regression analyses, age was not associated with survival outcomes (all $p>0.05$ ). In the TCGA cohort, patient with age $\leq 60$ years has $7 \%$ less DDR gene mutations $(p=0.59)$. We found higher age distribution in patients with luminal $(p<0.001)$ and luminal infiltrated $(p=0.002)$ compared to those with luminal papillary subtype.

Conclusions While younger patients may have less mutational tumor burden, our analysis failed to show an association of age with response to preoperative chemotherapy or survival outcomes. Therefore, the use of preoperative chemotherapy should be considered regardless of patient age.
\end{abstract}

Keywords Bladder cancer $\cdot$ Chemotherapy $\cdot$ Age $\cdot$ Response

\section{Introduction}

Neoadjuvant chemotherapy (NAC) followed by radical cystectomy (RC) and pelvic lymphadenectomy is the standard of care for muscle invasive bladder cancer (MIBC) [1].

While NAC has shown to improve survival [2-4], not every patient will respond to this preoperative therapy [5].

Shahrokh F. Shariat

sfshariat@gmail.com

Extended author information available on the last page of the article
Identification of patients who are unlikely to respond to NAC is of paramount importance for clinical decision making and patient counseling to avoid overtreatment and minimize unnecessary adverse events. This is specifically true for bladder cancer (BC) patients as they have, in general, various comorbidities and are often frail [6, 7]. While several factors, such as clinical tumor stage, histological variants, patient sex, exposure to carcinogens and tumor mutational burden haven been linked to the response to NAC [5, 8-15], only little is known about the association of age with response to and survival after 
NAC and RC. A recent analysis of The Cancer Genome Atlas (TCGA) showed an age-related distribution of total mutational burden, neoantigen load, molecular subtypes and intra-tumoral immune signaling in MIBC [16]. Based on these findings, we hypothesize that there might be an age-dependent response to preoperative chemotherapy.

To address this question, we analyzed the data originating from a multicenter cooperation on preoperative chemotherapy in RC.

\section{Materials and methods}

\section{Patient selection and intervention}

We retrospectively reviewed our multicenter database of 1543 patients treated with preoperative chemotherapy followed by RC and lymphadenectomy between 2000 and 2013.

Patients who received less than 2 cycles of preoperative chemotherapy $(n=151)$, those with unknown clinical stage $(n=24)$, those with unknown pathological stage $(n=53)$ and those who did not receive cisplatin-based combination chemotherapy $(n=210)$ were removed, leaving 1105 patients for final analyses (supplementary figure S1). No patient had clinically distant metastases on preoperative imaging.

Preoperative chemotherapy regimens consisted of methotrexate, vinblastine, doxorubicin, and cisplatin (MVAC), dose dense MVAC (ddMVAC) or gemcitabine and cisplatin (GemCis). The chemotherapy regimen and number of cycles were administered at clinician discretion and according to institutional standards.

All RC procedures were performed by an open technique. The decision for the type of urinary diversion was based on disease characteristics, patient wishes and performance status. All surgical specimens were processed according to standard pathologic procedures and staged according to the TNM classification.

\section{Outcome measurement}

Primary outcome of the study was pathologic objective response (pOR), defined as ypT0-Ta-Tis-T1N0. Secondary outcomes of the study were pathologic complete response (pCR), defined as ypT0N0, overall survival (OS) and cancerspecific survival (CSS).

Time-to-event was calculated from the administration of the first chemotherapy cycle until the last follow-up. Cause of death was recorded through patient chart review or death certificates [17]. We evaluated age as continuous variable and stratified the population based on age quartiles.

\section{Age and molecular landscape}

We used data from 395 TCGA patients [18] to investigate the prevalence of TCGA molecular subtypes (luminal papillary, luminal infiltrated, luminal, basal squamous and neuronal) and DNA damage repair (DDR) gene alterations according to patient age. We selected ERCC2, RB1, ATM, FANCC ATR, BRCA1, BRCA2, ERCC5, RAD51C, and REQLC4 as key DDR genes based on prior reports and current ongoing prospective trials $[14,19,20]$.

\section{Statistical analyses}

We compared the distribution of clinicopathologic features between age groups using the chi-square test for categorical variables and the Mann-Whitney-U test for continuous variables. We evaluated the association of patient age with pathologic response using univariable and multivariable logistic regression modeling. Due to the even distribution of the data between groups, adjustments using, i.e., propensity score were not applied.

We used two different approaches for the time-toevent analysis. First, we used the Cox regression analysis to investigate the association of age with OS and CSS. Survival functions were plotted using the Kaplan-Maier estimates. Second, we estimated the marginal probability of death from $\mathrm{BC}$ using competing risk analysis where death of other cause was considered the competing event. The proportional hazard was modeled using the Fine and Gray function.

We investigated the validity of the survival model testing the proportional hazard assumption and visually assessed the functional form of the association of age with cancer-specific death using the plot of Martingale residuals from a null Cox model against age.

Due to the exploratory character of the study, statistical significance was considered at $p<0.05$, but not in a confirmatory manner. Therefore, no adjustment for multiplicity was performed. Statistical analyses were performed with R (The R Project, Vienna, Austria).

\section{Results}

Overall, 437 (40\%) patients had a pOR and 234 (21\%) had pCR. There was no difference in clinicopathologic features, distribution in pOR or pCR between age quartiles (Table 1).

For the primary endpoint, on univariable logistic regression analysis, patient age was not associated with 
Table 1 Clinicopathologic features of 1,105 patients treated with neoadjuvant chemotherapy followed by radical cystectomy and lymphadenectomy for muscle-invasive bladder cancer, stratified by age quartiles

\begin{tabular}{|c|c|c|c|c|c|c|}
\hline Variable & Overall, $N=1105^{\mathrm{a}}$ & $27-57, N=280^{\mathrm{a}}$ & $58-64, N=288^{\mathrm{a}}$ & $65-71, N=290^{\mathrm{a}}$ & $72-87, N=247^{\mathrm{a}}$ & $p$-value \\
\hline Sex & & & & & & $>0.9$ \\
\hline Female & $258(23 \%)$ & $63(22 \%)$ & $70(24 \%)$ & $70(24 \%)$ & $55(22 \%)$ & \\
\hline Male & $847(77 \%)$ & $217(78 \%)$ & $218(76 \%)$ & $220(76 \%)$ & $192(78 \%)$ & \\
\hline cT stage & & & & & & $>0.9$ \\
\hline cT2 & $680(62 \%)$ & $170(61 \%)$ & $178(62 \%)$ & $180(62 \%)$ & $152(62 \%)$ & \\
\hline cT3 & $299(27 \%)$ & $75(27 \%)$ & $78(27 \%)$ & $80(28 \%)$ & $66(27 \%)$ & \\
\hline cT4 & $126(11 \%)$ & $35(12 \%)$ & $32(11 \%)$ & $30(10 \%)$ & $29(12 \%)$ & \\
\hline NAC regimen & & & & & & 0.11 \\
\hline ddMVAC & $139(13 \%)$ & $35(12 \%)$ & $45(16 \%)$ & $36(12 \%)$ & $23(9.3 \%)$ & \\
\hline GEM-CIS & $813(74 \%)$ & $195(70 \%)$ & $206(72 \%)$ & $220(76 \%)$ & $192(78 \%)$ & \\
\hline MVAC & $153(14 \%)$ & $50(18 \%)$ & $37(13 \%)$ & $34(12 \%)$ & $32(13 \%)$ & \\
\hline NAC cycles & & & & & & 0.11 \\
\hline $2-4$ & $1,028(93 \%)$ & $267(95 \%)$ & $260(90 \%)$ & $272(94 \%)$ & $229(93 \%)$ & \\
\hline 5 or more & $77(7.0 \%)$ & $13(4.6 \%)$ & $28(9.7 \%)$ & $18(6.2 \%)$ & $18(7.3 \%)$ & \\
\hline ypT stage & & & & & & 0.6 \\
\hline урТ0 & $251(23 \%)$ & $67(24 \%)$ & $63(22 \%)$ & $62(21 \%)$ & $59(24 \%)$ & \\
\hline ypT1-Ta-Tis & $226(20 \%)$ & $49(18 \%)$ & $72(25 \%)$ & $61(21 \%)$ & $44(18 \%)$ & \\
\hline урT2 & $203(18 \%)$ & $53(19 \%)$ & $45(16 \%)$ & $57(20 \%)$ & $48(19 \%)$ & \\
\hline урT3-T4 & $425(38 \%)$ & $111(40 \%)$ & $108(38 \%)$ & $110(38 \%)$ & $96(39 \%)$ & \\
\hline ypN stage & & & & & & 0.9 \\
\hline ypN0 & $818(74 \%)$ & $213(76 \%)$ & $213(74 \%)$ & $212(73 \%)$ & $180(73 \%)$ & \\
\hline ypN1 & $103(9.3 \%)$ & $24(8.6 \%)$ & $26(9.0 \%)$ & $27(9.3 \%)$ & $26(11 \%)$ & \\
\hline ypN2 & $153(14 \%)$ & $37(13 \%)$ & $37(13 \%)$ & $43(15 \%)$ & $36(15 \%)$ & \\
\hline ypN3 & $31(2.8 \%)$ & $6(2.1 \%)$ & $12(4.2 \%)$ & $8(2.8 \%)$ & $5(2.0 \%)$ & \\
\hline Variant histology & $99(9.0 \%)$ & $25(8.9 \%)$ & $21(7.3 \%)$ & $28(9.7 \%)$ & $25(10 \%)$ & 0.7 \\
\hline Lymph nodes removed & $19(12,28)$ & $19(13,28)$ & $18(11,28)$ & $19(13,28)$ & $19(12,27)$ & 0.6 \\
\hline STSM & & & & & & $>0.9$ \\
\hline Negative & $918(83 \%)$ & $233(83 \%)$ & $240(83 \%)$ & $241(83 \%)$ & $204(83 \%)$ & \\
\hline Positive & $81(7.3 \%)$ & $21(7.5 \%)$ & $22(7.6 \%)$ & $20(6.9 \%)$ & $18(7.3 \%)$ & \\
\hline Not evaluable & $106(9.6 \%)$ & $26(9.3 \%)$ & $26(9.0 \%)$ & $29(10 \%)$ & $25(10 \%)$ & \\
\hline pOR & $437(40 \%)$ & $112(40 \%)$ & $124(43 \%)$ & $110(38 \%)$ & $91(37 \%)$ & 0.5 \\
\hline $\mathrm{pCR}$ & $234(21 \%)$ & $65(23 \%)$ & $60(21 \%)$ & $57(20 \%)$ & $52(21 \%)$ & 0.8 \\
\hline
\end{tabular}

MVAC: methotrexate, vinblastine, doxorubicin, cisplatin; ddMVAC: dose dense methotrexate, vinblastine, doxorubicin, cisplatin; GEM-CIS: gemcitabine-cisplatin; STSM: soft tissue surgical margin; pOR: pathologic objective response; pCR: pathologic complete response

${ }^{a}$ Median (IQR) or Frequency (\%)

${ }^{b}$ Pearson's Chi-squared test; Kruskal-Wallis rank sum test

pOR (OR 1.00; 95\% CI 0.99-1.01; $p=0.7)$, pCR (OR $1.00 ; 95 \%$ CI $0.99-1.02 ; p=0.9)$ when evaluated as continuous variable. When the cohort was divided in quartiles, there was no association of patients age with outcomes (all $p>0.5$, supplementary Table $\mathrm{S} 1$ ).

Overall, 139 patients had insufficient follow-up, leaving 966 patients for survival analyses.

During a median follow-up for alive patients of 18 months (IQR 6-37), 303 (31\%) died of any cause and 250 (21\%) died of BC. On Cox regression analysis, age evaluated as continuous variable or stratified in quartiles was not associated with
CSS or OS (all $p>0.2$ ). Similarly, on competing risk regression analysis age was not associated with cancer-specific death (all $p>0.052$ ) (Fig. 1 and Table 2). The proportional hazard assumption was not violated $(p=0.72)$, confirming the validity of the model (Supplementary Figure S2a). The Martingale residuals plot did not show an association of age with cancer-specific death (Supplementary Figure S2b).

In a subgroup analysis of patients with pOR, age evaluated as continuous variable or stratified in quartiles was not associated with CSS, OS or cancer-specific death (Supplementary table S2). 

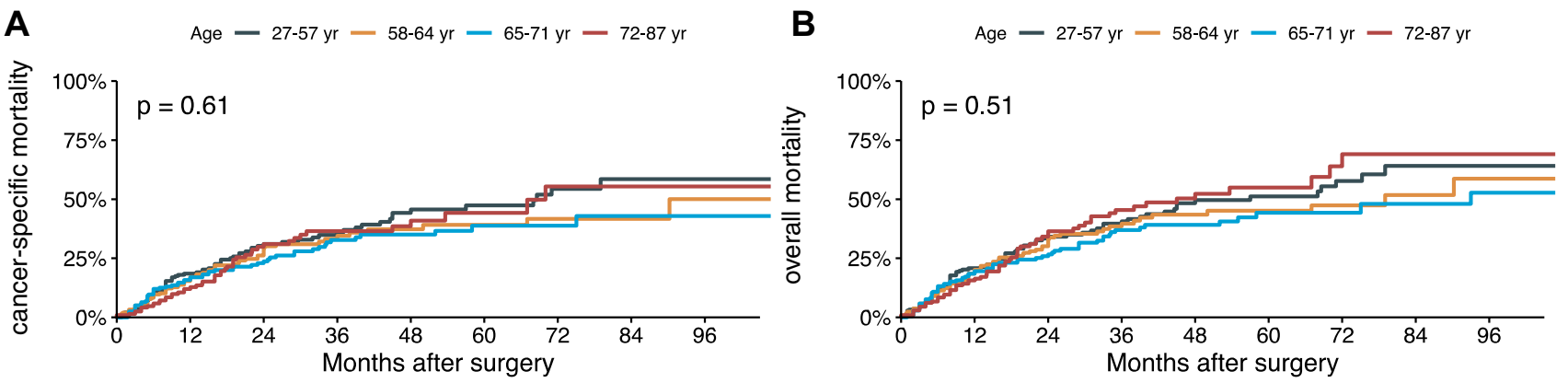

Number at risk

$\begin{array}{ccccccccc}27-57 \text { yr 248 } 146 & 85 & 61 & 39 & 28 & 18 & 8 & 4 \\ 58-64 \text { yr 253 } 151 & 96 & 53 & 36 & 27 & 21 & 10 & 6 \\ 65-71 \text { yr 252 } & 146 & 99 & 65 & 45 & 28 & 20 & 13 & 9 \\ 72-87 \text { yr 213 } & 117 & 59 & 41 & 26 & 12 & 7 & 3 & 2\end{array}$

Number at risk

$\begin{array}{lllllllll}27-57 \text { yr 248 } & 146 & 85 & 61 & 39 & 28 & 18 & 8 & 4 \\ 58-64 \text { yr 253 } 151 & 96 & 53 & 36 & 27 & 21 & 10 & 6 \\ 65-71 \text { yr 252 } & 146 & 99 & 65 & 45 & 28 & 20 & 13 & 9 \\ 72-87 \text { yr 213 } & 117 & 59 & 41 & 26 & 12 & 7 & 3 & 2\end{array}$

\section{Cumulative number of events}

$\begin{array}{llllllllll}27-57 \mathrm{yr} & 0 & 39 & 58 & 63 & 71 & 72 & 75 & 76 & 76 \\ 58-64 \mathrm{yr} & 1 & 36 & 54 & 59 & 61 & 62 & 63 & 63 & 64 \\ 65-71 \mathrm{yr} & 0 & 36 & 46 & 56 & 58 & 60 & 60 & 61 & 61 \\ 72-87 \mathrm{yr} & 2 & 20 & 40 & 44 & 46 & 47 & 49 & 49 & 49\end{array}$

Cumulative number of events

$\begin{array}{llllllllll}27-57 \mathrm{yr} & 1 & 45 & 66 & 73 & 81 & 82 & 85 & 87 & 87 \\ 58-64 \mathrm{yr} & 1 & 43 & 63 & 70 & 73 & 74 & 75 & 76 & 77 \\ 65-71 \mathrm{yr} & 0 & 42 & 53 & 65 & 67 & 70 & 70 & 71 & 72 \\ 72-87 \mathrm{yr} & 2 & 27 & 50 & 57 & 61 & 62 & 65 & 65 & 65\end{array}$

Fig. 1 Survival function estimating the cancer-specific mortality (A) and overall mortality (B) in 966 patients treated with preoperative chemotherapy and radical cystectomy for muscle-invasive bladder cancer, stratified by age

Table 2 Survival analyses investigating the association of age with cancer-specific survival (CSS), overall survival (OS) and death from bladder cancer in 966 patients treated with preoperative chemotherapy followed by radical cystectomy and lymphadenectomy

\begin{tabular}{|c|c|c|c|c|c|c|c|c|c|}
\hline & \multicolumn{3}{|c|}{ Overall survival } & \multicolumn{3}{|c|}{ Cancer-specific survival } & \multicolumn{3}{|c|}{ Fine and Gray model } \\
\hline & HR & $95 \% \mathrm{CI}$ & $p$-value & HR & $95 \% \mathrm{CI}$ & $p$-value & HR & $95 \% \mathrm{CI}$ & $p$-value \\
\hline Age (years) & 1.00 & $0.98,1.01$ & 0.6 & 0.99 & $0.98,1.00$ & 0.11 & 0.99 & $0.97-1.00$ & 0.052 \\
\hline \multicolumn{10}{|l|}{ Age groups } \\
\hline $27-57$ & - & - & & - & - & & & - & - \\
\hline $58-64$ & 0.89 & $0.66,1.21$ & 0.5 & 0.85 & $0.61,1.19$ & 0.3 & 0.83 & $0.59-1.16$ & 0.27 \\
\hline $65-71$ & 0.82 & $0.60,1.11$ & 0.2 & 0.80 & $0.57,1.12$ & 0.2 & 0.80 & $0.58-1.12$ & 0.20 \\
\hline $72-87$ & 1.01 & $0.74,1.40$ & $>0.9$ & 0.88 & $0.62,1.26$ & 0.5 & 0.81 & $0.57-1.15$ & 0.25 \\
\hline
\end{tabular}

HR: Hazard Ratio; CI: Confidence Interval
Analyzing the TCGA cohort, we found that 35\%, 42\%, $42 \%$ and $43 \%$ of patients aged $34-60,61-69,70-76$ and 77-90 years had at least one mutation in the selected DDR gene panel, respectively (Fig. $2 \mathrm{~A}, p=0.59$ ). When age was analyzed as a continuous variable, we found an association of ERCC2 mutations with older age (Fig. 2B). There was no difference in the distribution of age according to overall gene mutation (Fig. 2C, $p=0.13$ ) Finally, we found older age distribution in patients with luminal $(p<0.001)$ and luminal infiltrated $(p=0.002)$ compared to those with luminal papillary molecular subtype (Fig. 2D).

\section{Discussion}

We investigated the association of patient age with pathologic response and survival after preoperative chemotherapy using a large multicenter cohort and found no association of age evaluated as continuous variable or stratified in quartiles. Moreover, we found no association of age with CSS, OS or cancer-specific death.

The risk of BC incidence increases with age [21]. This is attributed to several factors including cumulative exposure 


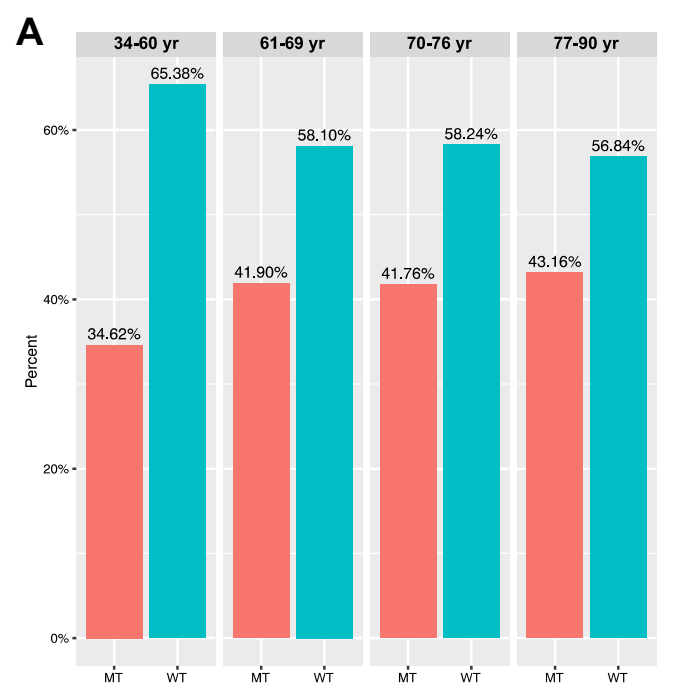

B
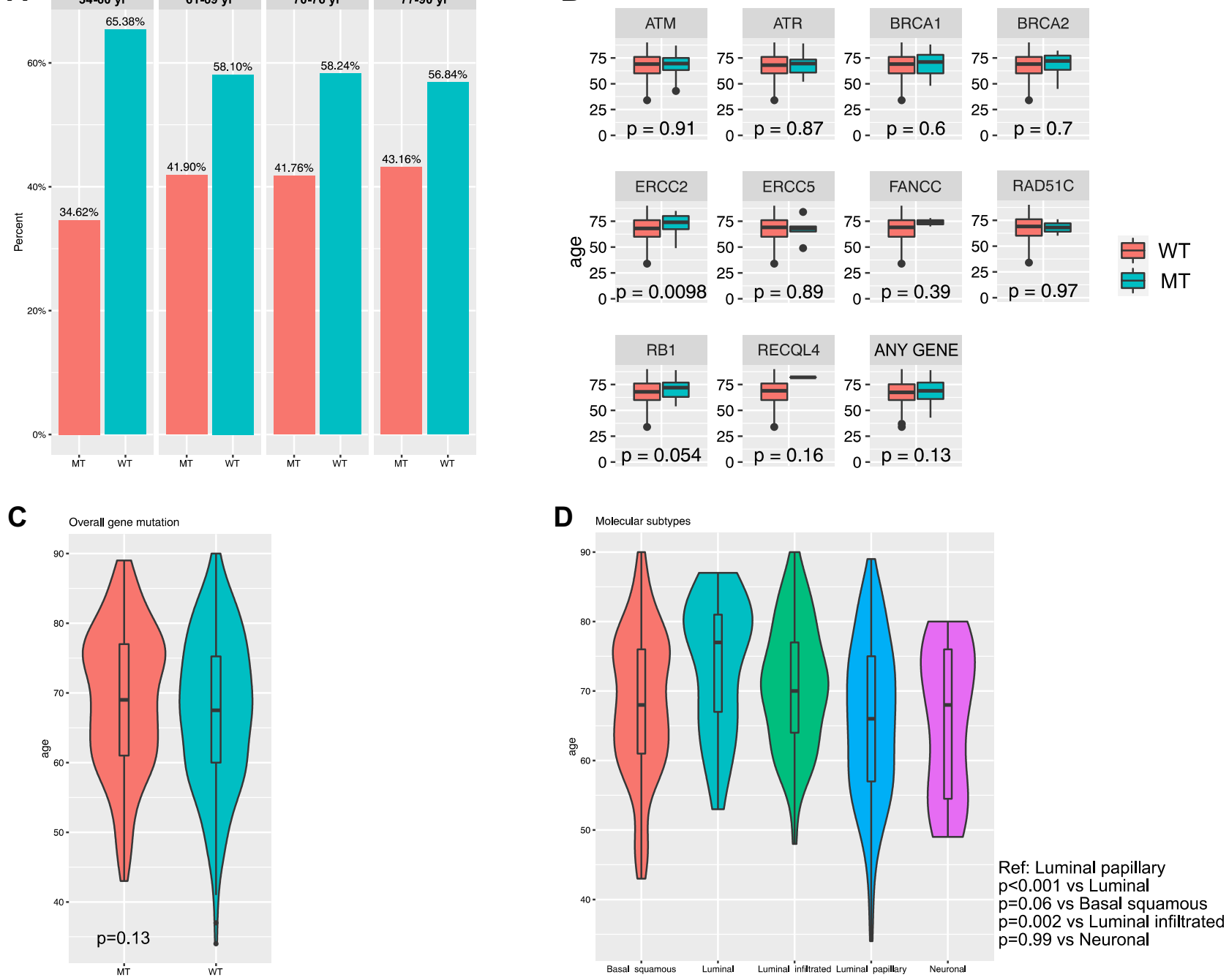

Fig. 2 A Prevalence of selected DNA damage repair (DDR) gene mutations in 395 patients with muscle-invasive bladder cancer stratified by age quartiles. B Distribution of selected DDR gene mutations according to age. C Distribution of selected DDR gene muta- tions according to age. D Distribution of mRNA cluster molecular subtypes according to age. Data extracted from The Cancer Genome Atlas [18]. WT: wild type; MT: mutated

The randomized SWOG-8710 trial compared the effect of NAC plus RC with RC alone in patients with MIBC [4]. The investigators performed subgroup analyses based on patient age using the median age of 64 years as the cut-off. They reported a median OS in patients $\leq 64$ years of 104 months compared to 61 months in patients $>64$ years $(p=0.05)$. In SWOG-8710, the association of pathologic response rates with age was not investigated. Pathologic response to chemotherapy is a generally accepted surrogate marker for survival in a trial level and could be influenced by patient age. In an analysis of 189 patients originating from the Retrospective International Study of Cancers of the Urothelial Tract (RISC), authors found an association between pathologic downstaging and response to chemotherapy with $\mathrm{RC}$ and preoperative chemotherapy are $>65$ years $[2,3]$. 
improved survival in MIBC. Authors performed an external validation of these finds in 2010 patients originating from the National Cancer Database (NCDB) [31]. In this report, age was included as continuous variable in the multivariable analysis and was significantly associated with outcomes in the NCDB cohort but not in the RISC cohort. In a multicenter retrospective study of 640 patients, authors aimed to identify prognostic features for cancer-specific death after NAC. While age was significantly associated with cancerspecific death on univariable Cox analysis, this association disappeared in the multivariable analysis and was, therefore, not included in the prognostic model [32]. Similarly, singlecenter series reported no difference in pathologic response or survival between younger and older patients [33, 34].

When interpreting these results, one could assume that the statistical non-significant association of age with outcomes is because retrospective studies are underpowered and prospective trials are not powered for the secondary endpoints. This can partially explain our findings that failed to show an association of age with oncologic outcomes. Indeed, the number of patients included in our study was probably too small to detect a significant difference between cohorts. Corroboration of these finding in a larger cohort could shed light on this.

Moreover, it should also be considered that the association of age with oncologic outcomes in MIBC is not only dependent on the biology of the disease itself, but also on barriers to access health care and sub-optimal treatment in older patients. Indeed, these patients may tend to receive less aggressive surgeries and sub-therapeutic dosing of systemic therapies [28, 29, 35-39]. Moreover, it has been shown that patients included in clinical trials only marginally reflect the real-world scenario. Indeed, only $15 \%$ of clinical trials could feasibly be replicated using currently available realworld data sources [40]. With this study, we complement the literature to help inform clinical practice, regarding patient selection and counseling.

The mutational landscape of UCB has been a particular focus of recent research. An analysis of the TGCA project has shown an association of somatic mutation rate with age in patients with MIBC [16]. This generates the hypothesis that there might be an age-dependent response to preoperative chemotherapy. We corroborated these findings with a granular analysis of the TCGA project investigating a panel of established DRR genes and molecular subtypes and their association with age. Younger patients had less DDR gene mutations while older patients had more luminal and luminal infiltrated molecular subtypes. Moreover, we found a significantly different distribution of age in patients with ERCC2 mutation. Molecular subtypes have been associated with response to preoperative chemotherapy and survival. Specifically, luminal infiltrated tumors showed lower response rates and survival while luminal papillary showed better survival rates, regardless of preoperative chemotherapy [25]. However, these findings need further exploration in welldesigned prospective clinical trials.

Despite all its strengths, our study is not devoid of limitations which are mainly inherent in its retrospective design. We had no information on smoking status, exposure to chemical compounds and renal function. Therefore, we could not adjust our analyses for the effect of these variables. We could not adjust for the effects of complications that occurred during preoperative chemotherapy resulting in subsequent dose reduction or sub-therapeutic dosing. There was no central pathological review of the specimens. The preoperative staging was not standardized and we had no information on preoperative comorbidities assessed by a validated score. Moreover, variability in standard practices, quality of surgery, frequency of surveillance imaging, sarcopenia and nutritional status in the elderly [41], as well as other selection biases and other not measurable confounders, may have influenced our results.

Development and identification of predictive and prognostic tools based on clinical variables and molecular biomarkers is essential for accurate identification of patients who are more likely to benefit from preoperative chemotherapy. In our analysis, we failed to prove an association of age with response to preoperative chemotherapy and survival in patients treated with RC. Our findings support the administration of preoperative chemotherapy, in patients who can tolerate it, regardless of their age. This hypothesis should be further investigated in prospective clinical trials and large, comparative retrospective cohorts.

\section{Conclusion}

This study found that age is not associated with response to preoperative chemotherapy. Therefore, it should be offered to patients regardless of age but rather based on their overall performance status and underlying renal function. While the TCGA analysis showed that younger patients may have less mutational tumor burden, this factor may not translate into response to preoperative chemotherapy and further research is needing into the impact of genetic factors and response to systemic therapy.

Supplementary Information The online version contains supplementary material available at https://doi.org/10.1007/s00345-021-03793-4.

Author contributions Conception and design: David D'Andrea, Peter C. Black, Shahrokh F. Shariat. Data acquisition: Peter C. Black, Homayoun Zargar, Kamran Zargar-Shoshtari, Adrian S. Fairey, Laura S. Mertens, Colin P. Dinney, Maria C. Mir, Laura-Maria Krabbe, Michael S. Cookson, Niels-Erik Jacobsen, Jeffrey S Montgomery, Nikhil Vasdev, Evan Y. Yu, Evanguelos Xylinas, Nicholas J. Campain, Wassim Kassouf, Marc A. Dall'Era, Jo-An Seah, Cesar E. Ercole, Simon Horenblas, Srikala S. Sridhar, John S. McGrath, Jonathan 
Aning, Jonathan L. Wright, Andrew C. Thorpe, Todd M. Morgan, Jeff M. Holzbeierlein, Trinity J. Bivalacqua, Scott North, Daniel A. Barocas, Yair Lotan, Petros Grivas, Andrew J. Stephenson, Jay B. Shah, Bas W. van Rhijn, Siamak Daneshmand, Philippe E. Spiess, Shahrokh F. Shariat. Analysis and interpretation of data: David D'Andrea, Peter C. Black, Shahrokh F. Shariat. Drafting of the manuscript: David D'Andrea, Peter C. Black, Shahrokh F. Shariat. Revision of the manuscript: Homayoun Zargar, Kamran Zargar-Shoshtari, Francesco Soria, Adrian S. Fairey, Laura S. Mertens, Colin P. Dinney, Maria C. Mir, Laura-Maria Krabbe, Michael S. Cookson, Niels-Erik Jacobsen, Jeffrey S. Montgomery, Nikhil Vasdev, Evan Y. Yu, Evanguelos Xylinas, Nicholas J. Campain, Wassim Kassouf, Marc A. Dall'Era, Jo-An Seah, Cesar E. Ercole, Simon Horenblas, Srikala S. Sridhar, John S. McGrath, Jonathan Aning, Jonathan L. Wright, Andrew C. Thorpe, Todd M. Morgan, Jeff M. Holzbeierlein, Trinity J. Bivalacqua, Scott North, Daniel A. Barocas, Yair Lotan, Petros Grivas, Andrew J. Stephenson, Jay B. Shah, Bas W. van Rhijn, Siamak Daneshmand, Philippe E. Spiess. Statistical analysis: David D'Andrea. Supervision: Peter C. Black, Shahrokh F. Shariat.

Funding Open access funding provided by Medical University of Vienna. Not applicable.

Availability of data and materials Data can be provided on request.

Code availability Programming codes can be provided on request.

\section{Declarations}

Conflict of interest Dr. D'Andrea has nothing to disclose. Dr. Black reports personal fees from AbbVie, Asieris, Astra-Zeneca, Astellas, Bayer, Biosyent, BMS, EMD-Serono, H3-Biomedicine, Janssen, Merck, Roche, Sanofi, Urogen, Ferring, TerSera, Pfizer, Decipher Biosciences, iProgen, Genentech, MDx Health outside the submitted work; In addition, Dr. Black shares a patent with Decipher Biosciences. Dr. Zargar has nothing to disclose. Dr. Zargar-Shoshtari has nothing to disclose. Dr. Soria has nothing to disclose. Dr Fairey has nothing to disclose. Dr. Mertens has nothing to disclose. Dr. Dinney has nothing to disclose. Dr Mir has nothing to disclose. Dr. Krabbe has nothing to disclose. Dr. Cookson reports personal fees from Myovant Sciences, Jannsen, Astellas, Bayer, Ferring outside of the submitted work. Dr. Jacobsen has nothing to disclose. Dr Montgomery has nothing to disclose. Dr. Vasdev has nothing to disclose. Dr. Yu reports personal fees from Amgen, personal fees from Astra-zeneca, grants and personal fees from Bayer, personal fees from Clovis, grants and personal fees from Dendreon, personal fees from Janssen, grants and personal fees from Merck, personal fees from Pharmacyclics, grants and personal fees from Seagen, outside the submitted work. Dr. Xylinas has nothing to disclose. Dr. Campain has nothing to disclose. Dr. Kassouf reports personal fees from Janssen, Ferring, Sesen bio, Merck, Roche, Astellas outside of the submitted work. Dr. Dall'Era has nothing to disclose. Dr. Seah has nothing to disclose. Dr. Ercole has nothing to disclose. Dr. Horenblas has nothing to disclose. Dr. Sridhar reports personal fees from Pfizer, Merck, Roche, BMS, Bayer, Astra-Zeneca, Astellas, Janssen, Immunomedex, grant from Bayer, Janssen, Pfizer outside of the submitted work. Dr. McGrath has nothing to disclose. Dr. Aning has nothing to disclose. Dr. Wright has nothing to disclose. Dr. Thorpe has nothing to disclose. Dr Morgan has nothing to disclose. Dr Holzbeierlein reports advisory board for Basilea outside of the submitted work. Dr. Bivalacqua has nothing to disclose. Dr. North has nothing to disclose. Dr. Barocas has nothing to disclose. Dr. Lotan reports advisory board or personal fees for C2I genomics, Photocure, Astra-Zeneca, Merck, Fergene, Abbvie, Cleveland Diagnostics, BMS, Nucleix, Abbott, Cepheid, Pacific Edge. FKD, MDxHealth, Biocan- cell, GenomeDx Biosciences, Storz, Vessi Medical, CAPs Medical outside of the submitted work. Dr. Grivas reports personal fees from Astra-Zeneca; Bayer; Bristol-Myers Squibb; Clovis Oncology; Dyania Health, Driver; EMD Serono; Exelixis; Foundation Medicine; Genentech/Roche; Genzyme; GlaxoSmithKline; Heron Therapeutics; Immunomedics, Janssen; Merck; Mirati Therapeutics; Pfizer; Seagen; QED Therapeutics. Research Funding to Institution from Merck; Pfizer, Clovis Oncology, Bavarian Nordic, Immunomedics, Debiopharm, BristolMyers Squibb, QED Therapeutics, GlaxoSmithKline, Kure It Cancer Research outside of the submitted work. Dr. Stephenson has nothing to disclose. Dr. Shah has nothing to disclose. Dr. Rhijn has nothing to disclose. Dr. Daneshmand reports personal fees from Janssen, Ferring, Photocure, Taris, Spectrum, Pacific Edge, QED, Abbvie, Johnson \& Johnson, Seagen, Nucleix, Aduro, BMS outside of the submitted work. Dr. Spiess has nothing to disclose. Dr. Shariat reports personal fees from Astellas, Astra-Zeneca, Bayer, BMS, Cepheid, Ferring, Ipsen, Janssen, Lilly, MSD, Olympus, Pfizer, Pierre Fabre, Roche, Sanochemia, Sanofi, Wolff, outside the submitted work; in addition he has following patents Method to determine prognosis after therapy for prostate cancer; Granted 2002-09-06, Method to determine prognosis after therapy for bladder cancer; Granted 2003-06-19, Prognostic methods for patients with prostatic disease; Granted 2004-08-05, Soluble Fas urinary marker for the detection of bladder transitional cell carcinoma; Granted 2010-07-20.

Consent for publication All authors give their consent to publish the article in the current form.

Consent to participate Not applicable.

Ethics approval Not applicable for retrospective deidentified data.

Open Access This article is licensed under a Creative Commons Attribution 4.0 International License, which permits use, sharing, adaptation, distribution and reproduction in any medium or format, as long as you give appropriate credit to the original author(s) and the source, provide a link to the Creative Commons licence, and indicate if changes were made. The images or other third party material in this article are included in the article's Creative Commons licence, unless indicated otherwise in a credit line to the material. If material is not included in the article's Creative Commons licence and your intended use is not permitted by statutory regulation or exceeds the permitted use, you will need to obtain permission directly from the copyright holder. To view a copy of this licence, visit http://creativecommons.org/licenses/by/4.0/.

\section{References}

1. Witjes JA, Bruins HM, Cathomas R et al (2020) European Association of Urology Guidelines on muscle-invasive and metastatic bladder cancer: summary of the 2020 guidelines. Eur Urol. https:// doi.org/10.1016/j.eururo.2020.03.055

2. Vale CL (2005) Neoadjuvant chemotherapy in invasive bladder cancer: update of a systematic review and meta-analysis of individual patient data advanced bladder cancer $(\mathrm{ABC})$ meta-analysis collaboration. Eur Urol 48:202-206. https://doi.org/10.1016/j. eururo.2005.04.006

3. Yin M, Joshi M, Meijer RP et al (2016) Neoadjuvant chemotherapy for muscle-invasive bladder cancer: a systematic review and two-step meta-analysis. Oncol 21:708-715. https://doi.org/ 10.1634/theoncologist.2015-0440

4. Grossman HB, Natale RB, Tangen CM et al (2003) Neoadjuvant chemotherapy plus cystectomy compared with cystectomy alone 
for locally advanced bladder cancer. New Engl J Med 349:859866. https://doi.org/10.1056/nejmoa022148

5. Zargar H, Espiritu PN, Fairey AS et al (2015) Multicenter assessment of neoadjuvant chemotherapy for muscle-invasive bladder cancer. Eur Urol 67:241-249. https://doi.org/10.1016/j.eururo. 2014.09.007

6. Chappidi MR, Kates M, Patel HD et al (2016) Frailty as a marker of adverse outcomes in patients with bladder cancer undergoing radical cystectomy. Urologic Oncol Seminars Orig Investig 34:256.e1-256.e6. https://doi.org/10.1016/j.urolonc.2015.12.010

7. Shariat SF, Milowsky M, Droller MJ (2009) Bladder cancer in the elderly. Urologic Oncol Seminars Orig Investig 27:653-667. https://doi.org/10.1016/j.urolonc.2009.07.020

8. D'Andrea D, Black PC, Zargar H et al (2020) Impact of sex on response to neoadjuvant chemotherapy in patients with bladder cancer. Urologic Oncol Seminars Orig Investig. https://doi.org/ 10.1016/j.urolonc.2020.01.010

9. Black AJ, Zargar H, Zargar-Shoshtari K et al (2019) The prognostic value of the neutrophil-to-lymphocyte ratio in patients with muscle-invasive bladder cancer treated with neoadjuvant chemotherapy and radical cystectomy. Urol Oncol 38:3.e17-3. e27. https://doi.org/10.1016/j.urolonc.2019.09.023

10. Krimphove MJ, Szymaniak J, Marchese M et al (2019) Sex-specific differences in the quality of treatment of muscle-invasive bladder cancer do not explain the overall survival discrepancy. Eur Urol Focus. https://doi.org/10.1016/j.euf.2019.06.001

11. Kluth LA, Black PC, Bochner BH et al (2015) Prognostic and prediction tools in bladder cancer: a comprehensive review of the literature. Eur Urol 68:238-253. https://doi.org/10.1016/j.eururo. 2015.01.032

12. Pietzak EJ, Zabor EC, Bagrodia A et al (2018) Genomic differences between "primary" and "secondary" muscle-invasive bladder cancer as a basis for disparate outcomes to cisplatin-based neoadjuvant chemotherapy. Eur Urol 75:231-239. https://doi.org/ 10.1016/j.eururo.2018.09.002

13. Kimura S, Iwata T, Abufaraj $M$ et al (2019) Impact of gender on chemotherapeutic response and oncologic outcomes in patients treated with radical cystectomy and perioperative chemotherapy for bladder cancer: a systematic review and meta-analysis. Clin Genitourin Canc 18:78-87. https://doi.org/10.1016/j.clgc.2019. 11.007

14. Plimack ER, Dunbrack RL, Brennan TA et al (2015) Defects in DNA repair genes predict response to neoadjuvant cisplatinbased chemotherapy in muscle-invasive bladder cancer. Eur Urol 68:959-967. https://doi.org/10.1016/j.eururo.2015.07.009

15. Cacciamani GE, Ghodoussipour S, Mari A et al (2020) Association between smoking exposure, neoadjuvant chemotherapy response and survival outcomes following radical cystectomy: systematic review and meta-analysis. J Urol. https://doi.org/10. 1097/ju.0000000000000813

16. Seiler R, Wyatt AW, Black PC (2019) Molecular landscape of carcinoma invading bladder muscle: does patient age matter?: Comment. Bju Int 124:719-721. https://doi.org/10.1111/bju.14802

17. Rink M, Fajkovic H, Cha EK et al (2012) Death certificates are valid for the determination of cause of death in patients with upper and lower tract urothelial carcinoma. Eur Urol 61:854-855. https://doi.org/10.1016/j.eururo.2011.12.055

18. Robertson AG, Kim J, Al-Ahmadie $\mathrm{H}$ et al (2017) Comprehensive molecular characterization of muscle-invasive bladder cancer. Cell 171:540-556.e25. https://doi.org/10.1016/j.cell.2017.09.007

19. Allen EMV, Mouw KW, Kim P et al (2014) Somatic ERCC2 mutations correlate with cisplatin sensitivity in muscle-invasive urothelial carcinoma. Cancer Discov 4:1140-1153. https://doi.org/ 10.1158/2159-8290.cd-14-0623

20. (n.d.) Alliance A031701: A Phase II Study of Dose-dense Gemcitabine Plus Cisplatin (ddGC) in Patients with Muscle-invasive
Bladder Cancer with Bladder Preservation for Those Patients Whose Tumors Harbor Deleterious DNA Damage Response (DDR) Gene Alterations. https://www.allianceforclinicaltrials inoncology.org/main/cmsfile?cmsPath=/Public/Annual\%20Mee ting/files/A031701-Iyer-May2019.pdf

21. Burger M, Catto JWF, Dalbagni G et al (2013) Epidemiology and risk factors of urothelial bladder cancer. Eur Urol 63:234-241. https://doi.org/10.1016/j.eururo.2012.07.033

22. Dobruch J, Daneshmand S, Fisch M et al (2016) Gender and bladder cancer: a collaborative review of etiology, biology, and outcomes. Eur Urol 69:300-310. https://doi.org/10.1016/j.eururo. 2015.08.037

23. Rink M, Crivelli JJ, Shariat SF et al (2015) Smoking and bladder cancer: a systematic review of risk and outcomes. Eur Urol Focus 1:17-27. https://doi.org/10.1016/j.euf.2014.11.001

24. Freedman ND, Silverman DT, Hollenbeck AR et al (2011) Association between smoking and risk of bladder cancer among men and women. JAMA 306:737-745. https://doi.org/10.1001/jama. 2011.1142

25. Seiler R, Ashab HAD, Erho N et al (2017) Impact of molecular subtypes in muscle-invasive bladder cancer on predicting response and survival after neoadjuvant chemotherapy. Eur Urol 72:544 554. https://doi.org/10.1016/j.eururo.2017.03.030

26. Zargar H, Zargar-Shoshtari K, Lotan Y et al (2016) Final pathological stage after neoadjuvant chemotherapy and radical cystectomy for bladder cancer-Does pT0 predict better survival than pTa/Tis/T1? J Urol 195:886-893. https://doi.org/10.1016/j.juro. 2015.10.133

27. Zargar-Shoshtari K, Zargar H, Lotan Y et al (2016) A multiinstitutional analysis of outcomes of patients with clinically node positive urothelial bladder cancer treated with induction chemotherapy and radical cystectomy. J Urol 195:53-59. https://doi.org/ 10.1016/j.juro.2015.07.085

28. Shariat SF, Sfakianos JP, Droller MJ et al (2010) The effect of age and gender on bladder cancer: a critical review of the literature. BJU Int. 105(3):300

29. Noon AP, Albertsen PC, Thomas F et al (2013) Competing mortality in patients diagnosed with bladder cancer: evidence of undertreatment in the elderly and female patients. Brit J Cancer 108:1534. https://doi.org/10.1038/bjc.2013.106

30. Chamie K, Hu B, White RWD, Ellison LM (2008) Cystectomy in the elderly: does the survival benefit in younger patients translate to the octogenarians? Bju Int 102:284-290. https://doi.org/10. 1111/j.1464-410x.2008.07636.x

31. Martini A, Jia R, Ferket BS et al (2019) Tumor downstaging as an intermediate endpoint to assess the activity of neoadjuvant systemic therapy in patients with muscle-invasive bladder cancer. Cancer 125:3155-3163. https://doi.org/10.1002/cncr.32169

32. Mir MC, Marchioni M, Zargar H et al (2020) Nomogram predicting bladder cancer-specific mortality after neoadjuvant chemotherapy and radical cystectomy for muscle-invasive bladder cancer: results of an International Consortium. Eur Urol Focus. https://doi.org/10.1016/j.euf.2020.07.002

33. Leone AR, Zargar-Shoshtari K, Diorio GJ et al (2017) Neoadjuvant chemotherapy in elderly patients with bladder cancer: oncologic outcomes from a single institution experience. Clin Genitourin Canc 15:e583-e589. https://doi.org/10.1016/j.clgc.2017. 01.014

34. Fairey AS, Daneshmand S, Quinn D et al (2013) Neoadjuvant chemotherapy with gemcitabine/cisplatin vs. methotrexate/vinblastine/doxorubicin/cisplatin for muscle-invasive urothelial carcinoma of the bladder: a retrospective analysis from the University of Southern California. Urol Oncol Seminars Orig Investig 31:1737-1743. https://doi.org/10.1016/j.urolonc.2012.07.005

35. Prout GR, Wesley MN, Yancik R et al (2005) Age and comorbidity impact surgical therapy in older bladder carcinoma patients: a 
population-based study. Cancer 104:1638-1647. https://doi.org/ 10.1002/cncr.21354

36. Nielsen ME, Shariat SF, Karakiewicz PI et al (2007) Advanced age is associated with poorer bladder cancer-specific survival in patients treated with radical cystectomy. Eur Urol 51:699-708. https://doi.org/10.1016/j.eururo.2006.11.004

37. Soria F, Moschini M, Korn S, Shariat SF (2016) How to optimally manage elderly bladder cancer patients? Transl Androl Urol. 5:683-691. https://doi.org/10.21037/tau.2016.04.08

38. D'Andrea D, Soria F, Zehetmayer S et al (2020) Comparative effectiveness of radical cystectomy and radiotherapy without chemotherapy in frail patients with bladder cancer. Scand J Urol. https://doi.org/10.1080/21681805.2019.1711160

39. Zaid HB, Patel SG, Stimson CJ et al (2013) Trends in the utilization of neoadjuvant chemotherapy in muscle-invasive bladder cancer: results from the National Cancer Database. Urology 83:75-80. https://doi.org/10.1016/j.urology.2013.07.072

40. Bartlett VL, Dhruva SS, Shah ND et al (2019) Feasibility of using real-world data to replicate clinical trial evidence. Jama Netw Open 2:e1912869. https://doi.org/10.1001/jamanetworkopen. 2019.12869

41. Stangl-Kremser MA, D'Andrea D et al (2018) Sarcopenia as a predictive factor for response to upfront cisplatin-based chemotherapy in patients with muscle-invasive urothelial bladder cancer. Urol Int 101:197-200. https://doi.org/10.1159/000489013

Publisher's Note Springer Nature remains neutral with regard to jurisdictional claims in published maps and institutional affiliations.

\section{Authors and Affiliations}

\section{David D'Andrea' - Peter C. Black ${ }^{2} \cdot$ Homayoun Zargar ${ }^{2,3} \cdot$ Kamran Zargar-Shoshtari $^{4,5} \cdot$ Francesco Soria $^{6}$. Adrian S. Fairey ${ }^{7} \cdot$ Laura S. Mertens $^{8} \cdot$ Colin P. Dinney $^{9} \cdot$ Maria C. Mir $^{10,11} \cdot$ Laura-Maria Krabbe $^{12,13}$. Michael S. Cookson ${ }^{14} \cdot$ Niels-Erik Jacobsen ${ }^{7}$. Jeffrey S. Montgomery ${ }^{15} \cdot$ Nikhil Vasdev $^{16,17} \cdot$ Evan Y. Yu $^{18}$. Evanguelos Xylinas $^{19}$. Nicholas J. Campain ${ }^{20}$. Wassim Kassouf ${ }^{21}$ - Marc A. Dall'Era ${ }^{22}$. Jo-An Seah ${ }^{23}$. Cesar E. Ercole ${ }^{10}$. Simon Horenblas ${ }^{8}$. Srikala S. Sridhar ${ }^{23}$. John S. McGrath ${ }^{20}$. Jonathan Aning ${ }^{20,24}$. Jonathan L. Wright ${ }^{25}$. Andrew C. Thorpe ${ }^{17}$. Todd M. Morgan ${ }^{15}$. Jeff M. Holzbeierlein ${ }^{26}$. Trinity J. Bivalacqua 27 . Scott North ${ }^{28,29}$. Daniel A. Barocas ${ }^{30}$ - Yair Lotan ${ }^{12}$. Petros Grivas ${ }^{18,31}$. Andrew J. Stephenson ${ }^{10}$. Jay B. Shah ${ }^{9,32}$. Bas W. van Rhijn ${ }^{8}$. Siamak Daneshmand ${ }^{33}$. Philippe E. Spiess ${ }^{4}$. Shahrokh F. Shariat ${ }^{1,12,34,35,36}$}

1 Department of Urology, Comprehensive Cancer Center, Medical University of Vienna, Währinger Gürtel 18-20, 1090 Vienna, Austria

2 Department of Urologic Sciences, University of British Columbia, Vancouver, BC, Canada

3 Department of Urology, Western Health, Melbourne, Australia

4 Department of Genitourinary Oncology, H Lee Moffitt Cancer Center and Research Institute, Tampa, FL, USA

5 University of Auckland, Auckland, New Zealand

6 Department of Urology, Molinette Hospital, University of Turin, Turin, Italy

7 University of Alberta, Edmonton, AB, Canada

8 Department of Urology, The Netherlands Cancer Institute - Antoni Van Leeuwenhoek Hospital, Amsterdam, The Netherlands

9 Department of Urology, MD Anderson Cancer Center, Houston, TX, USA

10 Glickman Urological and Kidney Institute, Cleveland Clinic, Cleveland, OH, USA

11 Department of Urology, Fundacion Instituto Valenciano de Oncologia, Valencia, Spain

12 Department of Urology, University of Texas Southwestern Medical Center, Dallas, TX, USA

13 Department of Urology, University of Münster, Münster, Germany

14 Department of Urology, University of Oklahoma College of Medicine, Oklahoma City, OK, USA
15 Department of Urology, University of Michigan Health System, Ann Arbor, MI, USA

16 Hertfordshire and Bedfordshire Urological Cancer Centre, Department of Urology, Lister Hospital, Stevenage, UK

17 Department of Urology, Freeman Hospital, Newcastle Upon Tyne, UK

18 Department of Medicine, Division of Medical Oncology, University of Washington School of Medicine and Fred Hutchinson Cancer Research Center, Seattle, WA, USA

19 Department of Urology, Hôpital Bichat-Claude Bernard, Assistance Publique-Hôpitaux de Paris, Université de Paris, Paris, France

20 Department of Surgery, Exeter Surgical Health Services Research Unit, Royal Devon and Exeter NHS Trust, Exeter, UK

21 Department of Surgery (Division of Urology), McGill University Health Center, Montreal, Canada

22 Department of Urology, Davis Medical Center, University of California At Davis, Sacramento, CA, USA

23 Princess Margaret Hospital, Toronto, ON, Canada

24 Bristol Urological Institute, North Bristol NHS Trust, Bristol, UK

25 Department of Urology, University of Washington, Seattle, WA, USA

26 Department of Urology, University of Kansas Medical Center, Kansas City, KS, USA 
27 Department of Urology, The James Buchanan Brady Urological Institute, The Johns Hopkins School of Medicine, Baltimore, MD, USA

28 Cross Cancer Institute, Edmonton, AB, Canada

Department of Oncology, University of Alberta, Edmonton, $\mathrm{AB}$, Canada

30 Department of Urologic Surgery, Vanderbilt University Medical Center, Nashville, TN, USA

31 Department of Hematology and Medical Oncology, Taussig Cancer Institute, Cleveland Clinic, Cleveland, USA

32 Department of Urology, Stanford University School of Medicine, Stanford, CA, USA
33 USC/Norris Comprehensive Cancer Center, Institute of Urology, University of Southern California, Los Angeles, CA, USA

34 Departments of Urology, Weill Cornell Medical College, New York, NY, USA

35 Department of Urology, Second Faculty of Medicine, Charles University, Prag, Czech Republic

36 Institute for Urology and Reproductive Health, I.M. Sechenov First Moscow State Medical University, Moscow, Russia 